\title{
On The Decipherment of Linear A Words Ku-Ro and Ki-Ro in the Common Kartvelian Language
}

\author{
Gia Kvashilava \\ Ivane Javakhishvili Tbilisi State University, Tbilisi, Georgia
}

\begin{abstract}
The paper presents the ancient Kartvelian scripts of Crete dated from the nineteenth to fifteenth century BC. On Linear A (LA) tablets mathematical data were written in the Common Kartvelian language.

The vast bulk of LA signs are graphically and phonetically identical to those of Mycenaean Linear $\mathrm{B}$ and the Cypriot syllabic scripts. The problem of the language of LA inscriptions has long remained unsolved, because it was supposed that the language and its culture no longer existed. The meanings of the LA inscriptions remained unidentified, and the LA tablets were declared to be written in an unknown dead language.

The LA word for addition ku-ro is especially interesting and important. „The meaning of one Linear A word is certain: ku-ro is the word which introduces totals, and must mean something like 'total' or 'so much'. If we could find such a word in a known language, the problem of Linear A might be solved "(J. Chadwick). The word ku-ro denoting arithmetic operation of addition, also the word kiro/*ki-lo (subtraction, lessening) are attested in all Kartvelian dialects: Georgian, Megrelian, Laz and Svan, which is shown in detail in the text of the paper.

Phonemic sequences of root+suffixal morphemes *kur-o(j) and *kir-o(j)/*kil-o(j) are the canonical forms of Common Kartvelian.

It is shown that the sequence root+suffix is regulated by the principle of monovocalism in Kartvelian dialects.

The study of Kartvelian material justifies the reliability of interpreting the LA script sign-sequences ku-ro and ki-ro/*ki-lo as the Common Kartvelian stems.
\end{abstract}

Keywords: Linear A script, Common Kartvelian dialects, principle of monovocalism. 